\title{
Clinical, ultrasound parameters and tumor marker-based mathematical models and scoring systems in pre-surgical diagnosis of adnexal tumors
}

\author{
Nabil Abdalla, Joanna Winiarek, Michał Bachanek, Krzysztof Cendrowski, Włodzimierz Sawicki \\ Chair and Clinic of Obstetrics, Gynecology and Oncology, Second Faculty of Medicine, Medical University of Warsaw, Poland
}

\begin{abstract}
The choice of management for patients with adnexal tumors requires careful pre-surgical assessment. In case of adnexal masses, the diagnostic difficulties arise from the heterogenic nature of the adnexal diseases, presence of multiple functional changes, and lack of early symptoms of malignancy. A reliable pre-surgical differentiation cannot be performed using clinical features, ultrasound examination, or tumor markers alone. New diagnostic techniques and novel markers are under investigations, however no single test can be used to conclusively differentiate between malignant and non-malignant adnexal masses. Mathematical models and scoring systems based on different clinical, ultrasonographic and laboratory parameters alone or together may facilitate the diagnosis. Selected mathematical models and scoring systems are presented in this article. Models using only ultrasound features include simple rules, regression models, Gynecologic Imaging Report and Data System, and various morphologic scores. Some logistic regression models are based on multiple clinical and ultrasound data. The OVA1 test is based on five tumor markers without using other data. The Risk of Malignancy Algorithm uses two tumor markers with one clinical parameter. i.e. the menopausal status. Some models used clinical, ultrasound and tumor marker data together. This group of models includes risk of malignancy indices, artificial neural networks, and the ADNEX model. Although some of these models have been compared in the literature, more prospective studies are needed to select the most effective model, to develop the existing models, or to create new more effective models of oncological assessment of the adnexal tumors.
\end{abstract}

Key words: ultrasonography, ovarian neoplasms, risk assessment, biomarkers

Ginekologia Polska 2016; 87, 12: 824-829

\section{INTRODUCTION}

Until today, no single imaging or laboratory test could be the source of a reliable differentiation between malignant and non-malignant adnexal tumors. The initial diagnosis of the adnexal masses depends on clinical examination, ultrasound assessment, and tumor marker levels. Subjective ultrasound assessment of the adnexal tumors is one of the basic methods used for their classification. However, result interpretation depends mainly on the experience of the examiner [1,2]. Thus, there exists a distinct need to establish certain criteria and definitions for ultrasound evaluation of the adnexa and their pathologies. In 2000, the International Ovarian Tumor Analysis (IOTA) group presented uniform terms and definitions for ultrasound description of the ad- nexal masses. The IOTA methodology enables ultrasound examiners to assess adnexal pathologies in the same way, independently of their experience [3]. Logistic regression models are systemic mathematical methods to establish an algorithm for detecting the likelihood of an occurrence of a certain event. They are already used in the management of adnexal masses to detect malignancy, using multiple different parameters to improve pre-surgical diagnostic differentiation of these pathologies. The final histological diagnosis of the adnexal tumors correlates with numerous parameters rather than with a single one. Forward stepwise selection of the parameters during model formation enables incorporation of the most important parameters into the equation of the logistic model. The result of these mod- 
els represents the likelihood of malignancy and can help choose the optimal method of adnexal tumor management, whether expectant or operative [4]. Precise pre-operative assessment is of great importance for patients in the reproductive age as in this case the surgical approach should consider fertility-sparing surgery for malignant diseases and minimal invasive surgery for non-malignant pathologies to preserve the ovarian reserve [5]. The choice of radical surgery is essential for patients with adnexal malignancies. Nowadays, laparoscopy can replace laparotomy in the treatment and staging of ovarian cancer [6]. In this group of patients, aseptic oncologic methods should be used to prevent iatrogenic rupture of adnexal malignant masses and dissemination of malignant cells [7]. Pre-surgical suspicion of malignant diseases enables examiners to refer patients to gynecologic oncology units without delay. The overall survival rate of patients treated in these units is higher than in non-oncologic units [8]. In this review article, we present selected mathematical models and scoring systems used for pre-surgical differentiation of adnexal tumors. Some of the parameters used in certain models e.g. papillary projection, are not compatible with the IOTA group definitions since these models had been introduced earlier. This may cause confusion as papillary projection cannot have the height of $<3 \mathrm{~mm}$ according to the IOTA group [3].

\section{ULTRASOUND PARAMETER-BASED MODELS}

\section{Simple rules}

In 2008, the IOTA group presented the simple rules method to classify adnexal masses pre-operatively. According to these rules, there are five malignant ultrasound features ( $M$ - malignant) and five benign features $(B-$ benign). Malignant ultrasound features include irregular solid tumor, ascites, at least four papillary projections, irregular multilocular-solid mass with a diameter of $\geq 100 \mathrm{~mm}$, intense vascularization of the mass assessed as grade 4 on the scale from 1 to 4 according to the IOTA terms and definitions. The following ultrasound features are considered as benign: unilocular cyst, solid areas with the maximum diameter of $<7 \mathrm{~mm}$, acoustic shadows, regular multilocular cyst with the maximum diameter of $<100 \mathrm{~mm}$, and absence of blood flow in color Doppler scan, i.e. grade 1 according to IOTA. The presence of at least one (M) malignant feature, in the absence of (B) benign features, will classify the mass as malignant. The presence of at least one (B) benign feature, with the absence of any (M) malignant features, enable the examiner to classify the mass as benign. In case of the presence of both, benign and malignant features, or when none of the them can be confirmed, the mass will be assessed as 'inconclusive' and will require further assessment by an expert. The sensitiv- ity and specificity of simple rules to detect malignancy in the study of the IOTA group were $95 \%$ and $91 \%$, respectively. Simple rules can be used by non-expert examiners to classify adnexal masses. However, approximately $25 \%$ of them will be considered as inconclusive and will need assessment by an ultrasound expert [2].

\section{Regression models}

In the study of Prompeler HJ et al., from 1997, an ultrasound-based regression model was presented. The following ultrasound parameters were included: ascites, solid areas without acoustic shadows, masses with at least 30\% solid area, tumor diameter, multilocular structures, and surface of the cyst. At cut-off level of $10 \%$, the sensitivity and specificity were $86.5 \%$ and $92.6 \%$, respectively for premenopausal and $93 \%$ and $82.7 \%$, respectively for postmenopausal patients [9].

\section{Gynecologic Imaging - Report and Data System}

In 2009, Amor F et al., presented an ultrasound-based Gynecologic Imaging — Report and Data System (GI-RADS), which has five grades of ultrasonographic assessment of the adnexa and their pathologies [10]. The system is based on the concept of Breast Imaging Report and Data System (BI-RADS). BI-RADS classification, used primarily for mammography assessment, is now used for the ultrasound evaluation of the breast. This classification system helps to communicate between sonographers and clinicians in the management of breast pathologies [11]. According to this system, normal ultrasound appearance of an ovary with no risk of malignancy is considered to be grade 1. The presence of functional changes, most probably benign, with malignancy probability of $<1 \%$, represents grade 2 . These changes include follicular cysts, corpora lutea, and hemorrhagic cysts. Grade 3 represents adnexal masses, probably benign, with the malignancy probability of $1-4 \%$. The following pathologies belong to this grade: endometriotic cysts, simple cysts, mature teratoma, hydrosalpinx, paraductal cysts, peritoneal pseudocysts, pedunculated fibroids, and inflammatory masses. Adnexal tumors, probably malignant, with the malignancy probability of $5-20 \%$ constitute grade 4 . This grade includes tumors which cannot be considered as grades 1-3, having one or two of the following malignant ultrasound features: papillary projections, septa, solid areas, ascites and vascularization within solid areas, papillary projections and central part of the solid tumors assessed in color or power Doppler examination. Adnexal masses, most probably malignant, which have at least three malignant features represent grade 5 . The risk of malignancy in this grade exceeds $20 \%[10,11]$. This system may help physicians to choose the most appropriate, grade-dependent method of management. Expectant management is the method of 
choice for grade 2 tumors. Grade 3 adnexal masses need surgical intervention by gynecologists, while grade 4-5 patients should be referred to gynecological oncologist for further management [11]. The sensitivity and specificity of GI-RADS in the study of Amor et al., were 92\% and 97\%, respectively [10].

\section{Morphologic scores}

Morphologic ultrasound features are used in these models. A certain value can be allocated for each ultrasound feature. The values of the parameters are summated and the sum represents the result of the morphologic model which can classify the adnexal mass as malignant or non-malignant at certain cut-off level [12-15]. Examples of morphological scores for the assessment of adnexal masses are shown in Table 1.

\section{CLINICAL DATA AND ULTRASOUND PARAMETER-BASED MODELS}

\section{Logistic regression models}

The model, proposed by Tailor et al., in 1997, included three parameters in the equation: age, time averaged maximum velocity (TAMXV), and presence or absence of papillary projections (values of 1 and 0 , respectively). The following equation is used: „probability of malignancy $=1 / 1+\mathrm{e}^{-z^{\prime \prime}}$, where $z=(0.1273 x$ age $)+(0.2794 x$ TAMXV $)+(4.4136 x$ papillary projection score) -14.2046 , and $e$ is the mathematical constant and the base value of the natural logarithm. A cut-off level at $50 \%$ was established for this model. The sensitivity and specificity in that study were $86.7 \%$ and $98.1 \%$, respectively [4].

The IOTA group suggested two logistic regression models in 2005. A total of 12 clinical and ultrasound parameters were used in the logistic regression model 1 (LR1). These parameters with the value allocated for each parameter in the final equation are presented in Table 2 [16].

The sensitivity and specificity of this model at cut-off level $10 \%$ reached $93 \%$ and $77 \%$, respectively. The probability of malignancy is calculated in the following equation: $y=1 /\left(1+e^{-z}\right)$, where $z=-6.7468$ $+1.5985(1)-0.9983(2)+0.0326(3)+0.00841(4)-0.8577(5)$ $+1.5513(6)+1.1737(7)+0.9281(8)+0.0496(9)+1.1421(10)$ $-2.3550(11)+0.4916(12)$ and $e$ is the mathematical constant and base value of natural logarithms. This model is the result of multi-center prospective studies [16].

The IOTA group modified the first logistic regression model and introduced the logistic regression model 2 (LR2), which is based only on 6 parameters. Clinical data included only (1) patient age. Ultrasound parameters included (2) ascites, (3) papillary projections with detectable blood flow, (4) maximum diameter of the largest solid area, (5) irregular cyst wall, and (6) acoustic shadows. Interpretation of these parameters with the values used for them in the equation is identical to Table 2 . For this simplified model, the following equation is used: $y=1 /\left(1+e^{-z}\right)$, where $z=-5.3718+0.0354(1)$ $+1.6159(2)+1.1768(3)+0.0697(4)+0.9586(5)-2.9486(6)$, $e$ is the mathematical constant and base value of natural logarithms. At cut-off level of $10 \%$, the sensitivity and specificity of this model reached $92 \%$ and $75 \%$, respectively [16].

\section{Serum tumor marker-based models}

A laboratory test, commercially known as OVA1, was cleared by the Food and Drug Administration (FDA) in 2009 [17]. This test uses an algorithm based on five tumor markers to predict adnexal malignancy. The following markers are used in this model: CA125, transferrin, transthyretin (prealbumin), apolipoprotein $A 1$ and $\beta 2$-microglobulin. The result of this test is calculated using the Ovacal program, which includes an algorithm for pre-surgical prediction of the presence of malignant ovarian disease. The result of the test has a range of $0-10$. High probability of malignancy is defined as a result $\geq 5$ for premenopausal and 4.4 for postmenopausal patients. The use of multiple tumor markers in this model increases the diagnostic performance of CA125 alone. The sensitivity and specificity of OVA1 test in the study of Ueland FR et al., in 2011 reached 93\% and 43\%, respectively [18].

\section{Clinical data and tumor marker-based models}

In 2009, Moore RG et al., presented the Risk of Malignancy Algorithm (ROMA) for the assessment of risk of malignancy of adnexal tumors. This algorithm is based on serum level of CA125 and Human Epididymis protein (HE4), taking into consideration the menopausal status of the patient. Predictive index (PI) for premenopausal patients is calculated in the following way: $\mathrm{PI}=-12.0+2.38^{*}$ $\mathrm{LN}(\mathrm{HE} 4)+0.0626^{*} \mathrm{LN}(\mathrm{CA} 125)$, while for postmenopausal patients: $\mathrm{PI}=-8.09+1.04 * \mathrm{LN}(\mathrm{HE} 4)+0.732 * \mathrm{LN}(\mathrm{CA} 125)$, where $L N$ is the natural logarithm. Predictive probability (PP) is calculated in the following way: $\mathrm{PP}=\exp (\mathrm{PI}) /[1-\exp (\mathrm{PI})]$. Cut-off levels used to define high risk of ovarian malignancy by Moore et al., are $13.1 \%$ and $27.7 \%$ for pre- and postmenopausal patients, respectively. Sensitivity and specificity of ROMA for premenopausal patients were $76.5 \%$ and $74.8 \%$, respectively, while for postmenopausal patients: $92.3 \%$ and $74.7 \%$, respectively. In their study, menopause was defined as amenorrhea for at least one year, history of bilateral ovariectomy, or age $>55$ years for patients who did not remember the date of the last menstrual period. The use of tumor markers without considering ultrasound parameters eliminated the problem of subjective assessment of ultrasound examiners and the need to consult experts during the assessment process [19]. 


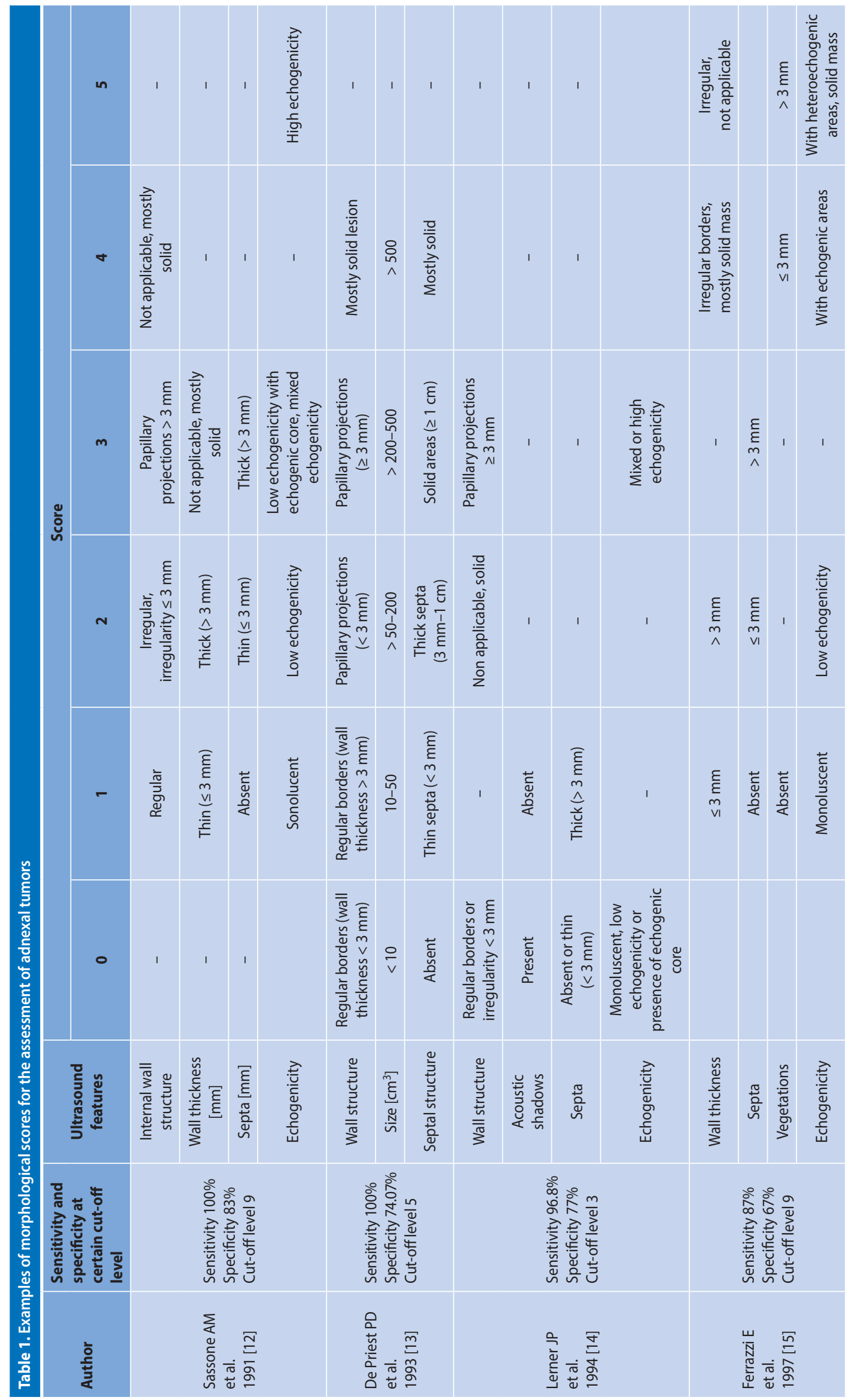


Table 2. Parameters used in the logistic regression model 1 proposed by the IOTA group with the interpretation of the data and values used in the formula

\begin{tabular}{l|c|c|}
\hline Examined parameter & $\begin{array}{c}\text { Interpretation } \\
\text { of the } \\
\text { parameters }\end{array}$ & $\begin{array}{c}\text { Value allocated } \\
\text { for the interpreted } \\
\text { parameters in the } \\
\text { final formula }\end{array}$ \\
\hline $\begin{array}{l}\text { (1) Personal history of } \\
\text { ovarian cancer }\end{array}$ & Yes & 1 \\
\hline (2) Current use of & No & 0 \\
\hline hormonal therapy & Yes & 1 \\
\hline (3) Age & No & 0 \\
\hline (4) Maximum diameter of \\
the tumor & - & Age in years \\
\hline (5) Pain during ultrasound & Yes & 1 \\
\hline examination & No & 0 \\
\hline of the tumor in mm
\end{tabular}

\section{CLINICAL DATA, ULTRASOUND PARAMETERS AND TUMOR MARKER LEVEL-BASED MODELS}

\section{Risk of malignancy indices}

A multimodal system based on ultrasound score $(U)$, menopausal status (M), and tumor marker CA125 was constructed by Jacobs et al. in 1990. The following ultrasound parameters are used during the ultrasound examination: presence of multilocular cyst, solid tumors, ascites, bilateral lesions, and metastases. For each ultrasound parameter a value of 1 was allocated. Ultrasound score $(U)$ equals 0 when there are no confirmed ultrasound features, $U=1$ when there is only one ultrasound feature, and $U=3$ when there are 2 to 5 features. Menopausal score $(M)$ is 1 for premenopausal pa- tients and 3 for postmenopausal patients. Risk of Malignancy Index (RMI I) is calculated according to the following formula: RMI I $=\mathrm{U} \times \mathrm{M} \times \mathrm{CA} 125$. Serum level of CA125 $(\mathrm{U} / \mathrm{mL})$ is used directly in the formula. When the cut-off level of RMI I was set at 200, the sensitivity and specificity in differentiating between malignant and non-malignant masses in this study were $85 \%$ and $97 \%$, respectively [20].

In 1996, Tinglustad S et al., modified RMI I and presented RMI II. These authors used the same formula, modifying only the values used for the $\mathrm{U}$ and $\mathrm{M}$ scores. The $\mathrm{U}$ score has a value of 1 , if there are no ultrasound features or when there is only one ultrasound feature. At least two features will give a value of $U=4$. An $M$ value of 1 was given for premenopausal and 4 for postmenopausal patients. In that study, the same cut-off level, i.e. 200, was used, resulting in the sensitivity and specificity of $80 \%$ and $92 \%$, respectively [21].

In 1999, Tinglustad S et al., yet again modified the formula and presented RMI III. The U score has a value of 1 when there is only one ultrasound feature and a value of 3 when there are at least two confirmed features. The M score had values identical to RMI I. The same cut-off level was used, with the sensitivity and specificity for differentiating between malignant adnexal tumors reaching $71 \%$ and $92 \%$, respectively [22].

In 2009, RMI was modified by Yamamato $Y$ et al., who presented RMI IV. A new element in the form of the maximum diameter $(\mathrm{S})$ of the mass was used in this modification. The $U$ and $M$ scores had values identical to those used in RMI II. The S parameter had a value of 1 when the maximum diameter of the mass was $<70 \mathrm{~mm}$, and $\mathrm{S}=2$ when the maximum diameter was $\geq 70 \mathrm{~mm}$. RMI IV was calculated using the formula: RMI IV $=U \times M \times C A 125 \times S$. Using this method at cut-off level of 450 , the sensitivity and specificity were $86.8 \%$ and $91 \%$, respectively [23].

\section{Artificial neural networks (ANN)}

Artificial neural networks (ANN) can be described as systems which change information, in the form of numerical values, through the synapses. This analytic model was presented by Timmerman D et al. in 1999. The following parameters were used in the first variant of ANN (ANN1): papillary projections, blood flow, CA125 level and the menopausal status, while in the second version (ANN2) the following parameters were considered: papillary projections, smooth surface, unilocular cyst, ascites, bilateral lesions, tumor marker CA125 and the menopausal status. The probability of malignancy is calculated using sophisticated mathematical models structured by the authors of that study. In ANN1, the probability of malignancy $>45 \%$ revealed the sensitivity and specificity of $87.5 \%$ and $92.7 \%$, respectively, while in ANN2 the probability of $>60 \%$ resulted in the sensitivity and specificity of $93.8 \%$ and $95.1 \%$, respectively [24]. 


\section{Prediction model}

In 2014, the IOTA group presented the Assessment of Different NEoplasias in the adneXa (ADNEX). This method can predict the probability of malignancy in five main categories (benign, borderline, stage I malignant disease, stage II-IV malignant disease and metastases). This model is based on serum CA125 levels, two clinical parameters and six ultrasound parameters. Clinical data include patient age and type of medical facility where the ultrasound was performed (oncological or not). Ultrasound features include maximum diameter of the lesion, maximum diameter of the largest solid area, presence of multilocular cyst with more than 10 locules, number of papillary projections, acoustic shadows and ascites. For cut-off level of $10 \%$, the sensitivity and specificity were $96.5 \%$ and $71.3 \%$, respectively. Precise prediction of adnexal tumor type helps to choose the most appropriate method of management. In this model, CA125 level may be omitted if not available, however this can bias the result, since CA125 is one of the most important predicting factors in the model. The ADNEX model, simple rules and the logistic regression models of the IOTA group are available at www.iotagroup. org and as mobile applications [25].

\section{SUMMARY}

In this article, we presented mathematical models and scoring systems which are used to calculate the probability of malignancy in patients with adnexal tumors [26]. Despite a great number of models which are proposed by different medical centers, until today there exists no single model or system which can conclusively discriminate between malignant and non-malignant adnexal tumors. Introduction of uniform definitions by the IOTA group enables examiners anywhere in the world to perform the examination in a systemic way, independently of the experience of the examiners. Also, a uniform ultrasound description of the adnexal tumors allows for result comparison between different centers in the world.

\section{Acknowledgment}

This study was supported by a grant project of the Medical University of Warsaw for research and scientific work aimed at scientific development of young doctors and $\mathrm{PhD}$ students (No. 2WA/PM21D/13).

\section{REFERENCES}

1. Van Gorp T, Veldman J, Van Calster B, [et al.]. Subjective assessment by ultrasound is superior to the risk of malignancy index (RMI) or the risk of ovarian malignancy algorithm (ROMA) in discriminating benign from malignant adnexal masses. Eur J Cancer. 2012, 48, 1649-1656.

2. Timmerman D, Testa AC, Bourne T, [et al.]. Simple ultrasound-based rules for the diagnosis of ovarian cancer. Ultrasound Obstet Gynecol. 2008, 31, 681-690.

3. Timmerman $\mathrm{D}$, Valentin $\mathrm{L}$, Bourne $\mathrm{TH}$, [et al.]. International Ovarian Tumor Analysis (IOTA) Group. Terms, definitions and measurements to describe the sonographic features of adnexal tumors: a consensus opinion from the International Ovarian Tumor Analysis (IOTA) Group. Ultrasound Obstet Gynecol. 2000, 16, 500-505.

4. Tailor A, Jurkovic D, Bourne $\mathrm{TH}_{\text {, }}$ [et al.]. Sonographic prediction of malignancy in adnexal masses using multivariate logistic regression analysis. Ultrasound Obstet Gynecol. 1997, 10, 41-47.

5. Spinelli C, Pucci V, Strambi S, [et al.]. Treatment of ovarian lesions in children and adolescents: a retrospective study of 130 cases. Pediatr Hematol Oncol. 2015, 32, 199-206.

6. Koo YJ, Kim JE, Kim YH, [et al.]. Comparison of laparoscopy and laparotomy for the management of early-stage ovarian cancer: surgical and oncological outcomes. J Gynecol Oncol. 2014, 25, 111-117.

7. Watanabe E, Tanaka K, Takeda N, [et al.]. Surgical technique to prevent spillage of cyst fluid during operation for cystic ovarian tumors. Pediatr Surg Int. 2013, 29, 645-649.

8. Woo YL, Kyrgiou M, Bryant A, [et al.]. Centralisation of services for gynaecological cancers - a Cochrane systematic review. Gynecol Oncol. 2012, 126, 286-290.

9. Prömpeler HJ, Madjar H, Sauerbrei W, [et al.]. Diagnostic formula for the differentiation of adnexal tumors by transvaginal sonography. Obstet Gynecol. 1997, 89, 428-433.

10. Amor F, Vaccaro H, Alcázar JL, [et al.]. Gynecologic imaging reporting and data system: a new proposal for classifying adnexal masses on the basis of sonographic findings. J Ultrasound Med. 2009, 28, 285-291.

11. Amor F, Alcázar JL, Vaccaro H, [et al.]. GI-RADS reporting system for ultrasound evaluation of adnexal masses in clinical practice: a prospective multicenter study. Ultrasound Obstet Gynecol. 2011, 38, 450-455.

12. Sassone AM, Timor-Tritsch IE, Artner A, [et al.]. Transvaginal sonographic characterization of ovarian disease: evaluation of a new scoring system to predict ovarian malignancy. Obstet Gynecol. 1991, 78, 70-76.

13. DePriest PD, Shenson D, Fried A, [et al.]. A morphology index based on sonographic findings in ovarian cancer. Gynecol Oncol. 1993, 51, 7-11.

14. Lerner JP, Timor-Tritsch IE, Federman A, Abramovich G. Transvaginal ultrasonographic characterization of ovarian masses with an improved, weighted scoring system. Am J Obstet Gynecol. 1994, 170, 81-85.

15. Ferrazzi E, Zanetta G, Dordoni D, [et al.]. Transvaginal ultrasonographic characterization of ovarian masses: comparison of five scoring systems in a multicenter study. Ultrasound Obstet Gynecol. 1997, 10, 192-197.

16. Timmerman D, Testa AC, Bourne T, [et al.]. International Ovarian Tumor Analysis Group. Logistic regression model to distinguish between the benign and malignant adnexal mass before surgery: a multicenter study by the International Ovarian Tumor Analysis Group.J Clin Oncol. 2005, 23, 8794-801.

17. U.S. Food and Drug Administration. FDA news release: FDA clears a Test for Ovarian Cancer. Test can help identify potential malignancies, guide surgical decisions. http://www.fda.gov/NewsEvents/Newsroom/PressAnnouncements/ucm182057.htm

18. Ueland FR, Desimone CP, Seamon LG, [et al.]. Effectiveness of a multivariate index assay in the preoperative assessment of ovarian tumors. Obstet Gynecol. 2011, 117, 1289-1297.

19. Moore RG, McMeekin DS, Brown AK, [et al.]. A novel multiple marker bioassay utilizing HE4 and CA 125 for the prediction of ovarian cancer in patients with a pelvic mass. Gynecol Oncol. 2009, 112, 40-46.

20. Jacobs I, Oram D, Fairbanks J, [et al.]. A risk of malignancy index incorporating CA 125, ultrasound and menopausal status for the accurate preoperative diagnosis of ovarian cancer. Br J Obstet Gynaecol. 1990, 97, 922-929.

21. Tingulstad S, Hagen B, Skjeldestad FE, [et al.]. Evaluation of a risk of malignancy index based on serum CA125, ultrasound findings and menopausal status in the pre-operative diagnosis of pelvic masses. $\mathrm{Br}$ J Obstet Gynaecol. 1996, 103, 826-831.

22. Tingulstad S, Hagen B, Skjeldestad FE, [et al.]. The risk-of-malignancy index to evaluate potential ovarian cancers in local hospitals. Obstet Gynecol. 1999, 93, 448-452.

23. Yamamoto $\mathrm{Y}$, Yamada R, Oguri $\mathrm{H}$, [et al.]. Comparison of four malignancy risk indices in the preoperative evaluation of patients with pelvic masses. Eur J Obstet Gynecol Reprod Biol. 2009, 144, 163-167.

24. Timmerman D, Verrelst $\mathrm{H}$, Bourne $\mathrm{TH}$, [et al.]. Artificial neural network models for the preoperative discrimination between malignant and benign adnexal masses. Ultrasound Obstet Gynecol. 1999, 13, 17-25.

25. Van Calster B, Van Hoorde K, Valentin L, [et al.]. Evaluating the risk of ovarian cancer before surgery using the ADNEX model to differentiate between benign, borderline, early and advanced stage invasive, and secondary metastatic tumours: prospective multicentre diagnostic study. BMJ. 2014, 349, g5920.

26. Michalak M, Gasiorowska E, Nowak-Markwitz E. Diagnostic value of CA125, HE4, ROMA and logistic regression model in pelvic mass diagnostics - our experience. Ginekol Pol. 2015, 86, 256-261. 